\title{
Article
}

\section{The Effects of Running Compared with Functional High-Intensity Interval Training on Body Composition and Aerobic Fitness in Female University Students}

\author{
Yining Lu ${ }^{1}$, Huw D. Wiltshire ${ }^{1}$, Julien S. Baker ${ }^{2}$ (D) and Qiaojun Wang ${ }^{3, *}$ \\ 1 Cardiff School of Sport and Health Sciences, Cardiff Metropolitan University, Cardiff CF5 2YB, UK; \\ st20184530@outlook.cardiffmet.ac.uk (Y.L.); hwiltshire@cardiffmet.ac.uk (H.D.W.) \\ 2 Department of Sport, Physical Education and Health, Hong Kong Baptist University, \\ Kowloon Tong, Hong Kong; jsbaker@hkbu.edu.hk \\ 3 Faculty of Sport Science, Ningbo University, Ningbo 315000, China \\ * Correspondence: wangqiaojun@nbu.edu.cn; Tel.: +86-138-0588-5586
}

\section{check for} updates

Citation: Lu, Y.; Wiltshire, H.D.; Baker, J.S.; Wang, Q. The Effects of Running Compared with Functional High-Intensity Interval Training on Body Composition and Aerobic

Fitness in Female University Students Int. J. Environ. Res. Public Health 2021, 18, 11312. https://doi.org/10.3390/ ijerph182111312

Academic Editors: Filipe Manuel Clemente and Ana Filipa Silva

Received: 13 October 2021

Accepted: 25 October 2021

Published: 28 October 202

Publisher's Note: MDPI stays neutral with regard to jurisdictional claims in published maps and institutional affiliations.

Copyright: (C) 2021 by the authors Licensee MDPI, Basel, Switzerland. This article is an open access article distributed under the terms and conditions of the Creative Commons Attribution (CC BY) license (https:/ / creativecommons.org/licenses/by/ $4.0 /)$.

\begin{abstract}
High-intensity interval running (HIIT-R) and high-intensity functional training (HIFT) are two forms of HIIT exercise that are commonly used. The purpose of this study was to determine the effects of HIFT on aerobic capacity and body composition when compared to HIIT-R in females. Twenty healthy, untrained female university students (age $20.5 \pm 0.7$ year) were randomly assigned to a 12-week HIIT-R or HIFT intervention. The HIIT-R group involved a $30 \mathrm{~s}$ maximal shuttle run with a 30 s recovery period, whereas the HIFT involved multiple functional exercises with a 2:1 work-active recovery ratio. Body composition, $\mathrm{VO}_{2} \max$, and muscle performance were measured before and post intervention. As a result, HIIT-R and HIIT-F stimulated similar improvements in $\mathrm{VO}_{2} \max (17.1 \% \pm 5.6 \%$ and $12.7 \% \pm 6.7 \%$, respectively, $p>0.05)$. Only the HIIT-F group revealed significant improvements in muscle performance (sit-ups, $16.5 \% \pm 3.1 \%$, standing broad jump $5.1 \% \pm 2.2 \%, p<0.05)$. Body fat percentage decreased $(17.1 \% \pm 7.4 \%$ and $12.6 \% \pm 5.1 \%$, respectively, $p<0.05)$ in both HIIT-R and HIIT-F with no between-group differences. We concluded that HIFT was equally effective in promoting body composition and aerobic fitness compared to HIIT-R. HIFT resulted in improved muscle performance, whereas the HIIT-R protocol demonstrated no gains.
\end{abstract}

Keywords: high-intensity interval training; high-intensity functional training; body composition; aerobic fitness; muscle performance

\section{Introduction}

Regular physical activity (PA) is beneficial for health [1-3]. Despite the well documented benefits of moderate- to vigorous-intensity PA, 31\% of adults worldwide do not engage in sufficient PA for health benefits as recommended by the World Health Organization (WHO) and the American College of Sports Medicine (ACSM) [4-6]. Frequently reported barriers to physical activity are physical exertion, time, and financial expenditure $[7,8]$. Thus, compared to traditional continuous training, which is characterized by long-duration, continuous aerobic exercises, and moderate-intensities, high-intensity interval training (HIT) appears to be an efficient pathway to enhance PA and improve health [9].

HIIT involves repeated bouts of high-intensity exercises separated by a recovery using low-intensity activities or inactivity [10]. Recent studies had indicated that HIIT has a similar, or even greater positive, effect on physical fitness, especially on body composition and cardiorespiratory health [11-14]. From a time/benefit perspective, HIIT appears to help physically inactive individuals overcome a major time and participation barrier to maintaining a healthier lifestyle [15]. 
Originally, HIIT was used to improve the performance of endurance athletes [16]. Cycling, running, and rowing are traditional exercise modalities that adopted the use of HIIT protocols, while for individuals who perform exercise for health and recreation, these traditional modalities seem boring and do not engage individuals because of the repetitive nature of the exercise combined with repetition. This is considered as a negative impact for maintaining regular exercise and has been cited as "lack of enjoyment" when investigating barriers to exercise [17].

The intrinsic factors of participants are also important when considering exercise adherence $[18,19]$. Several studies have revealed that adherence is affected by exercise intensity, especially among inactive individuals [20,21].

High-intensity functional training (HIFT) has become a relatively popular training modality in recent years and is an alternative to traditional aerobic activities. The HIFT protocol consists of a variety of functional movements that are executed at a high intensity $[22,23])$. Recently, several investigators have studied the effects of HIFT on physical fitness promotion. After engaging in HIFT protocols, participants show significant improvements in cardiorespiratory fitness $[24,25]$ and body composition $[25,26]$. Providing similar or greater health promotions compared to moderate-intensity continuous training, HIFT demonstrates further improvements in muscle fitness $[27,28]$. Additionally, participants perceive this type of activity to be more enjoyable when engaging in HIFT compared to those individuals performing traditional HIIT $[29,30]$. Moreover, most HIFT protocols are executed using the participant's own body weight, allowing the participant to control the exercise intensity. This helps to improve exercise adherence $[7,19,20]$.

Although studies have shown that HIFT has similar or superior benefits for physical fitness compared to moderate-intensity continuous training and have indicated more enjoyment compared to HIIT, the question remains as to whether HIFT is as efficient as HIIT for improving health-related fitness.

While HIFT is not synonymous with HIIT, they share an important conceptual commonality in the modality of both being of a high intensity. The current study was undertaken to clarify how a functional exercise based on HIIT would improve fitness parameters such as fat mass, blood pressure, $\mathrm{VO}_{2} \mathrm{max}$, and muscle endurance following a 12-week intervention compared to changes achieved using a running-based HIIT. The purpose of this study was to investigate the effects of different kinds of training on fitness parameters in untrained female university students. It was hypothesized that (a) aerobic fitness would be increased in both the HIIT-F and HIIT-R groups; (b) that fat mass would be decreased in both the HIIT-F and HIIT-R groups; and (c) that muscular strength and endurance would be improved in the HIIT-F group.

\section{Materials and Methods}

\subsection{Participants}

Twenty untrained healthy females who were physical inactive volunteered to participate the study. Participants who did not exercise for more than $2 \mathrm{~h}$ weekly for at least 12 months were considered as physically inactive [31]. All of the participants were in their second year of a non-physical education-related degree at Ningbo University. Similar self-reported menstrual cycles were required, ensuring the simultaneity of testing and training. Interventions were suspended for 1 week during menstruation, and the normal menstruation period lasted for 3 to 10 days [32,33]. A randomized controlled research design was utilized, and participants were randomly assigned into a running-based HIIT (HIIT-R) $(n=10)$ or a functional training-based HIIT (HIIT-F) $(n=10)$. The participants were nonsmokers and were instructed to maintain their normal dietary intake and lifestyle habits (sleep, sit, and physical activity) throughout the intervention. Nutritional supplements and intense exercise beyond their usual exercise habits were forbidden during the intervention period [31]. All of the participants were fully familiarized with the test procedures and data collection methods prior to the intervention. Written informed consent was provided 
by all participants. The study was approved by the Ningbo University ethics committee. The characteristics of the participants at baseline are detailed in Table 1.

Table 1. Baseline characteristics of HIIT-R and HIIT-F group.

\begin{tabular}{cccc}
\hline Parameter & HIIT-R Group $(\boldsymbol{n = 1 0 )}$ & HIIT-F Group $(\boldsymbol{n = 1 0 )}$ & $p$-Value \\
\hline Age $(\mathrm{yrs})$ & $20.7 \pm 0.6$ & $20.2 \pm 0.7$ & $p=0.14$ \\
Height $(\mathrm{m})$ & $161.1 \pm 3.1$ & $160.7 \pm 2.8$ & $p=0.76$ \\
Weight $(\mathrm{kg})$ & $56.6 \pm 6.7$ & $57.8 \pm 6.7$ & $p=0.69$ \\
Lean muscle mass $(\mathrm{kg})$ & $36.5 \pm 1.7$ & $36.0 \pm 2.1$ & $p=0.53$ \\
BMI $\left(\mathrm{kg} / \mathrm{m}^{2}\right)$ & $21.9 \pm 3.1$ & $22.4 \pm 2.2$ & $p=0.70$ \\
WHR & $0.80 \pm 0.0$ & $0.80 \pm 0.0$ & $p=0.79$ \\
Body fat (\%) & $31.6 \pm 4.1$ & $32.3 \pm 3.6$ & $p=0.71$ \\
HR resting $(\mathrm{bpm})$ & $70.8 \pm 13.9$ & $72.5 \pm 11.2$ & $p=0.77$ \\
HR max $(\mathrm{bpm})$ & $188.2 \pm 9.7$ & $189.1 \pm 10.4$ & $p=0.18$ \\
VO2max $(\mathrm{mL} / \mathrm{kg} / \mathrm{min})$ & $31.3 \pm 7.0$ & $32.8 \pm 5.4$ & $p=0.61$ \\
\hline
\end{tabular}

Notes: BMI, body mass index; bpm, beats per minute, HIIT-F, functional exercise-based high-intensity interval training; HIIT-R, running-based high-intensity interval training; HR resting, resting heart rate; HR max, maximal heart rate; $\mathrm{VO}_{2} \max$, maximal oxygen uptake; WHR, waist to hip ratio; yrs, years old.

\subsection{Procedures}

A randomized controlled trial was used in this study. Each participant completed twelve weeks of 36 sessions of HIIT-R or HIIT-F intervention (three sessions per week) comprising a total of $19 \mathrm{~min}$ per session (10 min warm-up, 4 min work-out, and $5 \mathrm{~min}$ cool-down). All sessions were conducted and monitored at the same indoor stadium and at the same time of day between 9:00-10:00 a.m. Heart rates (HR) were collected with an activity wristband (Mi Smart Band 5, Xiaomi, Beijing, China) during each session to ensure that the required high intensity was achieved. The reliability and validity of the heart rate index and distance index were reported in a previous study [34]. The activity wristband was required to be worn tightly on the participant's wrist. The HR index was measured based on changes in the light transmittance caused by blood flow density using optical sensing technology, and the distance index was measured by a triaxial acceleration sensor. Two measurement time points (pre- and post-intervention) were included. The participants were instructed to abstain from drugs, alcohol, and intense exercise two days prior to the baseline and post-intervention measurements. On the first measurement day, the participants presented themselves at 8:00 a.m. and underwent a body composition analysis, physical, and physiology measures as well as resting heart rate (HRresting) and blood pressure (BP) measurements under standardized conditions. The aerobic fitness assessment was conducted using a 12 min running test, which was completed on two days, with $24 \mathrm{~h}$ observed between each test. The first running test was scheduled on the first measurement day following the completion of all of the other tests, and the second trial was $24 \mathrm{~h}$ later. The average of the two data sets was used to assess aerobic fitness. After resting for a week [35], both groups began the training intervention. Post-intervention measurements were performed using the same methodologies as at baseline and were undertaken two days following all of the training sessions [11]. During the intervention period, additional exercises including habitual training were suspended.

\subsection{Physical, Physiological and Body Composition Assessment}

Participants were instructed to arrive at the laboratory 9:00 a.m. after a normal breakfast. Before the measurements were taken, participants were asked to empty their bladder to minimize measurement errors caused by "electrically silent" [36]. Under the guidance of two skilled operators and while wearing normal PE clothing, the participants stood on a bioelectrical impedance analysis device (BIA) (MC-180, TANITA CO., Dongguan, China) and data were presented from the device's associated software and included height, weight, waist and hip circumference, lean muscle mass, and body fat percentage. Body mass index 
(BMI) was obtained by dividing weight $(\mathrm{kg})$ by height $(\mathrm{m})$ squared. Waist-to-hip ratio (WHR) was obtained by dividing waist $(\mathrm{cm})$ by hip $(\mathrm{cm})$. Blood pressure and resting HR were measured using an automatic upper arm blood pressure monitor (HEM-1000, Omron, Dalian, China). The average of the two data sets was used for analysis.

\subsection{Aerobic Fitness Test}

The most reliable and effective way to measure aerobic capacity is to record each individual subject's $\mathrm{VO}_{2} \max$ [37]. Although maximal-effort tests are commonly used to measure $\mathrm{VO}_{2}$ max, for untrained participants, submaximal exercises can be used as a reliable measure to estimate this value. Cooper's 12 min running test was used to assess aerobic fitness in this study. All of the participants completed two trials of the running test separated by $24 \mathrm{~h}$ of rest. After a 5 min warm up, the participants were required to wear an activity wristband (Mi Smart Band 5, Xiaomi, China) and commenced running on a standard 400-metre running track. Subjects were instructed to run as many laps as possible on a standard outdoor track during the 12 min test period. All of the participants were encouraged verbally and were instructed to focus on their own pace throughout the test. The experimenter verbally provided the elapsed time at 3,6, and $9 \mathrm{~min}$. At the end of the 12 min period, the experimenter called "stop". All of the participants ceased running and stood still, until the distance achieved, and maximal heart rate (HRmax) were recorded. The HRmax displayed on the activity wristband was recorded immediately upon the cessation of exercise, and the higher value of the two trials was used for analysis. The total distance run was determined by measures obtained from the activity band. An estimated $\mathrm{VO}_{2} \mathrm{max}$ was calculated using Cooper's standardized equation [38]. The calculated $\mathrm{VO}_{2}$ max was highly correlated with the laboratory-determined one and had acceptable reliability and validity $(r=0.897)$ [38]. The average of the two data sets was used to determine the $\mathrm{VO}_{2} \max$.

\subsection{Muscle Performance Test}

Muscle performance was assessed using a field-based muscle fitness test battery. Timed sit-ups, push-ups with flexed knee (modified for females), and standing broad jump were recommended by previous studies to assess muscle performance [26,39-41]. All of the participants were instructed to perform the tests under supervision, and the data were recorded by the same experimenter. To assess abdominal muscular performance, the participants were asked to perform as many sit-ups as possible during a one-minute test period. The number of sit-ups that were completed correctly were recorded. A sit-up that met the following criteria was recorded: the participant lay supine on the mat with their hands crossed behind their head, elbows pointed straight forward, and knees bent at 90 degrees. The ankles were firmly held by the experimenter. During the execution of the test, the participants sat up with their heads clasped in their hands, and then their elbows touched or went over the knees, and the participant went back with their shoulders touching the mat [42]. To assess upper body strength and endurance, the flexed knee push-up option was used as a gender modification [39]. A correct flexed knee push-up met the following criteria: participants knelt on the mat with their knees bent to the mat with their arms propped on the mat slightly wider than the shoulders. When the test began, the participants were instructed to lower their body by bending their arms until their elbows were bent at a 90-degree angle and their chest was placed within 2 inches of the mat, subjects then pushed up to the starting position $[43,44]$. The number of correctly completed push-ups during a one-minute test period was recorded as upper body strength and endurance. Finally, the standing broad jump test was used to assess the muscle power of the lower limbs. The participants wore sneakers and stood behind the starting line with their feet placed naturally at a shoulder width apart. When testing began, the participants were instructed to bend the knees, swing the arms, and jump with both feet at the same time [45]. The jumping distance measured in centimeters was recorded, and the best of 
three jumps was used to determine lower limb performance. All scores were compared for statistical analysis.

\subsection{Intervention}

Exercise interventions commenced one week after the last measurement day. Both the HIIT-R and HIIT-F interventions were conducted three days per week on Mondays, Wednesdays, and Saturdays for twelve weeks. If the participants were unable to attend a scheduled exercise day, the exercise was performed on the next day and was monitored by the same researcher.

Participants in the HIIT-R group were required to complete 144 repetitions of maximal shuttle running for a total exercise time of $72 \mathrm{~min}$. Each bout included a $30 \mathrm{~s}$ maximal shuttle run between cones placed $20 \mathrm{~m}$ apart with a $30 \mathrm{~s}$ recovery period between runs. The validity and reliability of $40 \mathrm{~m}$ maximal shuttle run as a measure of anaerobic performance has been reported previously [46]. The participants completed 4 bouts per session over three sessions per week. Prior to the intervention, a familiarization trial was provided to acquaint the participants with the training procedure. Running and recovery times were recorded manually using a digital stopwatch by the same experimenter. Participants were encouraged to run at their individual maximal speed for each bout.

Participants in the HIIT-F group performed multiple functional exercises using their own body weight based on Tabata training [47]. According to a recent study [48], eight movements were implemented in each session (Table 2). Participants were motivated to complete as many repetitions of a given movement as possible over $20 \mathrm{~s}$ followed by a $10 \mathrm{~s}$ recovery in the form of low intensity stepping. There was no rest period between each movement. The total training time for each session was $4 \mathrm{~min}$.

Table 2. Details of the functional high-intensity interval training intervention.

\begin{tabular}{|c|c|c|c|}
\hline Duration & Frequency & Exercises & $\begin{array}{c}\text { Exercise Bout/ } \\
\text { Recovery Duration }\end{array}$ \\
\hline \multirow{16}{*}{12 weeks } & \multirow{16}{*}{3 sessions/week } & Jumping Jacks & $20 \mathrm{~s}$ \\
\hline & & Stepping & $10 \mathrm{~s}$ \\
\hline & & High knees & $20 \mathrm{~s}$ \\
\hline & & Stepping & $10 \mathrm{~s}$ \\
\hline & & Side to side squat & $20 \mathrm{~s}$ \\
\hline & & Stepping & $10 \mathrm{~s}$ \\
\hline & & Mountain climbers & $20 \mathrm{~s}$ \\
\hline & & Stepping & $10 \mathrm{~s}$ \\
\hline & & $\begin{array}{l}\text { Forearm plank to } \\
\text { high plank }\end{array}$ & $20 \mathrm{~s}$ \\
\hline & & Stepping & $10 \mathrm{~s}$ \\
\hline & & Burpees & $20 \mathrm{~s}$ \\
\hline & & Stepping & $10 \mathrm{~s}$ \\
\hline & & Deep squat jumps & $20 \mathrm{~s}$ \\
\hline & & Stepping & $10 \mathrm{~s}$ \\
\hline & & Butt kickers & $20 \mathrm{~s}$ \\
\hline & & Stepping & $10 \mathrm{~s}$ \\
\hline
\end{tabular}

The training frequency was the same as the HIIT-R group. All training exercises were recorded by video, which was provided to the HIIT-F participants prior to intervention to ensure that they were familiar with the movements and procedures. This video was played on a screen during the training intervention to ensure that the participants kept up with the rhythm of each movement. 
To ensure that the interventions were performed at adequate exercise intensity, participants' HRs were recorded throughout the session with an activity wristband. The peak heart rate (HR peak) of each session was considered to be $75 \%$ or more of the HRmax that had been recorded during Cooper's 12 min running test. All of the sessions began with a standardized $10 \mathrm{~min}$ low-to-moderate running and stretching followed by maximal shuttle run or functional training and ended with a 5 min cool-down and stretching.

\subsection{Statistical Analyses}

Statistical analyses were performed using SPSS, version 23.0 (Chicago, IL, USA). Data were presented as means $\mathrm{x} \pm \mathrm{SD}$. A two-factor analysis of variance with repeated measures was used to analyze differences in body composition, muscle performance, and aerobic capacity, with intervention (pretraining and post training) as a within-group factor and group (HIIT-R and HIIT-F) as a between-group factor. A significant intervention $\mathrm{x}$ group interaction was used to identify training-induced changes in body composition, muscle performance and aerobic capacity. Data were subsequently checked by Tukey's post hoc test if a significant interaction was revealed. Furthermore, paired t-tests were used to estimate within-group effects, and independent $t$-tests were conducted to examine differences between groups. The significance level was established as $p<0.05$.

\section{Results}

All of the participants completed all of the sessions over the twelve-week period. There were no significant between-group differences in the variables measured at baseline (Table 1).

\section{- Body Composition}

Body composition data are presented in Table 3. There was a significant decrease $(17.4 \% \pm 7.4 \%$ for HIIT-R and $12.6 \% \pm 5.1 \%$ for HIIT-F, $p<0.05)$ in the percent body fat for both groups (Figure 1a), with no interaction effect between HIIT-R and HIIT-F ( $p>0.05)$. Body mass index (BMI) (Figure 1b) and waist hip ratio (WHR) (Figure 1c) did not change in either intervention $(p>0.05)$. Lean muscle mass increased in both groups $(1.8 \% \pm 1.4 \%$ for HIIT-R and $1.2 \% \pm 1.2 \%$ for HIIT-F, $p<0.05$ ).

- Resting Heart Rate and Blood Pressure

Resting HR $(p<0.05)$ was improved compared to baseline in both intervention groups, while no interaction effect was observed. Resting systolic BP and diastolic BP remained unchanged $(p>0.05)$ after training in both the HIIT-R and HIIT-F groups.

- Aerobic Capacity

$\mathrm{VO}_{2} \max$ data was calculated from the following Cooper's equation: $\mathrm{VO}_{2} \max$ $(\mathrm{mL} / \mathrm{kg} / \mathrm{min})=($ distance $(\mathrm{m})-506) / 45 . \mathrm{VO}_{2} \max$ data for all participants are presented in Table 3. A significant increase $(p<0.05)$ in the $\mathrm{VO}_{2}$ max was demonstrated in both training groups compared to baseline measures, while no significant intervention $\mathrm{x}$ group interaction was revealed between HIIT-R and HIIT-F after intervention compared to baseline (Figure 1c). $(p>0.05)$. The extent of the change in $\mathrm{VO}_{2}$ max was $17.1 \% \pm 5.6 \%$ and $12.7 \% \pm 6.7 \%$ in the HIIT-R and HIIT-F groups, respectively.

\section{- $\quad$ Muscle Performance}

A significant intervention $x$ group interaction displayed significant changes in the HIIT-R and HIIT-F groups in terms of measures of abdominal and lower limb strength (Figure 1d). In the HIIT-F group, repetitions completed during the one-minute sit-up test increased $(p<0.05)$ by $16.5 \% \pm 3.1 \%$ and the distance obtained in the stand broad jumping test improved $(p<0.05)$ by $5.1 \% \pm 2.2 \%$, whereas these variables were unaltered $(p>0.05)$ in the HIIT-R group. Flexed push-ups were unaltered in both the HIIT-R and HIIT-F groups (Table 4). 
Table 3. Body composition and aerobic capacity data from HIIT-R and HIIT-F groups.

\begin{tabular}{|c|c|c|c|c|c|c|c|c|c|c|}
\hline \multirow[b]{2}{*}{ Parameter } & \multicolumn{4}{|c|}{ HIIT-R Group $(n=10)$} & \multicolumn{4}{|c|}{ HIIT-F Group $(n=10)$} & \multicolumn{2}{|c|}{$\begin{array}{c}\text { Interaction } \\
\text { Effect }\end{array}$} \\
\hline & Baseline & Post & $\Delta$ & $p$-Value & Baseline & Post & $\Delta$ & $p$-Value & $p$-Value & $\eta^{2}$ \\
\hline Weight (kg) & $56.6 \pm 6.7$ & $55.8 \pm 6.5$ & $-1.3 \% \pm 2.1 \%$ & ns & $57.8 \pm 6.7$ & $56.6 \pm 6.4$ & $-1.9 \% \pm 3.0 \%$ & ns & ns & 0.020 \\
\hline $\begin{array}{l}\text { Lean muscle } \\
\text { mass }(\mathrm{kg})\end{array}$ & $36.5 \pm 1.7$ & $37.2 \pm 1.8$ & $1.8 \% \pm 1.4 \%$ & $p<0.05$ & $36.0 \pm 2.1$ & $36.4 \pm 2.1$ & $1.2 \% \pm 1.2 \%$ & $p<0.05$ & ns & 0.056 \\
\hline BMI $\left(\mathrm{kg} / \mathrm{m}^{2}\right)$ & $21.9 \pm 3.1$ & $21.6 \pm 3.1$ & $-1.3 \% \pm 2.1 \%$ & ns & $22.4 \pm 2.2$ & $21.9 \pm 2.1$ & $-1.9 \% \pm 3.0 \%$ & ns & ns & 0.018 \\
\hline WHR & $0.8 \pm 0.0$ & $0.8 \pm 0.0$ & $-0.6 \% \pm 0.9 \%$ & ns & $0.8 \pm 0.0$ & $0.8 \pm 0.0$ & $-0.3 \% \pm 0.5 \%$ & ns & ns & 0.032 \\
\hline Body fat (\%) & $31.6 \pm 4.1$ & $26.3 \pm 4.8$ & $-17.1 \% \pm 7.4 \%$ & $p<0.01$ & $32.3 \pm 3.6$ & $28.3 \pm 3.9$ & $-12.6 \% \pm 5.1 \%$ & $p<0.01$ & ns & 0.118 \\
\hline $\begin{array}{l}\text { HR resting } \\
\quad(\mathrm{bpm})\end{array}$ & $76.5 \pm 10.1$ & $74.2 \pm 7.4$ & $-2.5 \% \pm 5.5 \%$ & ns & $77.8 \pm 9.1$ & $75.3 \pm 8.6$ & $-3.1 \% \pm 5.3 \%$ & ns & ns & 0.001 \\
\hline HR max (bpm) & $188.7 \pm 6.7$ & $185.8 \pm 6.0$ & $-1.5 \% \pm 1.1 \%$ & $p<0.05$ & $183.7 \pm 9.3$ & $181.8 \pm 7.8$ & $-1.0 \% \pm 1.3 \%$ & $p<0.05$ & ns & 0.050 \\
\hline $\begin{array}{c}\mathrm{VO} 2 \mathrm{max} \\
(\mathrm{mL} / \mathrm{kg} / \mathrm{min})\end{array}$ & $31.3 \pm 7.0$ & $36.7 \pm 8.8$ & $17.1 \pm 5.6 \%$ & $p<0.01$ & $32.8 \pm 5.4$ & $36.9 \pm 6.4$ & $12.7 \% \pm 6.7 \%$ & $p<0.01$ & ns & 0.075 \\
\hline
\end{tabular}

Note: $\Delta$ (post-baseline)/baseline; ns, no significance; partial $\eta^{2}$ value for effect size.

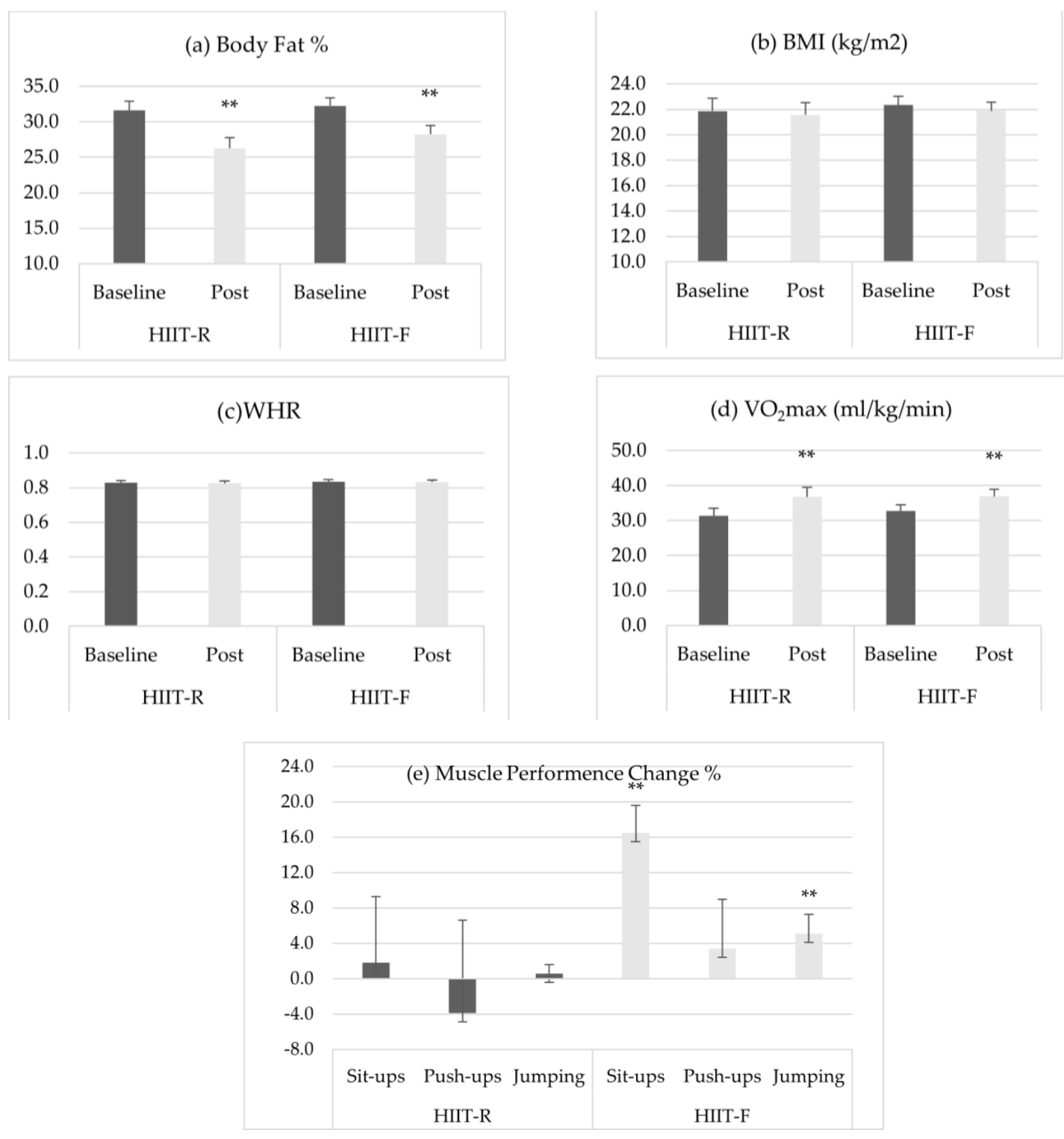

Figure 1. Changes in (a) BMI, (b) body fat \%, (c) WHR, (d) VO2max, and (e) muscle performance change. Note: ** significantly different from baseline at $p<0.01$. 
Table 4. Muscle performance data from HIIT-R and HIIT-F groups.

\begin{tabular}{ccccccccccc}
\hline & \multicolumn{3}{c}{ HIIT-R Group $(n=10)$} & & & \multicolumn{2}{c}{ HIIT-F Group $(n=10)$} & & Interaction Effect \\
\hline Parameter & Baseline & $\begin{array}{c}\text { Post- } \\
\text { Training }\end{array}$ & $\boldsymbol{\Delta}$ & $p$-Value & Baseline & $\begin{array}{c}\text { Post- } \\
\text { Training }\end{array}$ & $\Delta$ & $p$-Value & $p$-Value & $\eta^{2}$ \\
\hline $\begin{array}{c}\text { Sit-ups } \\
\text { (reps) }\end{array}$ & $35.3 \pm 6.7$ & $35.7 \pm 5.9$ & $1.8 \% \pm 7.5 \%$ & ns & $37.3 \pm 4.8$ & $43.4 \pm 5.3$ & $16.5 \% \pm 3.1 \%$ & $p<0.01$ & $p<0.01$ & 0.760 \\
$\begin{array}{c}\text { Flexed } \\
\text { push-ups } \\
\text { (reps) } \\
\begin{array}{c}\text { Standing } \\
\text { broad } \\
\text { jump }(\mathrm{cm})\end{array}\end{array}$ & $7.7 \pm 1.3$ & $7.4 \pm 1.6$ & $-3.9 \% \pm 10.5 \%$ & ns & $8.0 \pm 1.4$ & $8.3 \pm 1.7$ & $3.4 \% \pm 5.6 \%$ & ns & ns & 0.180 \\
\hline
\end{tabular}

Note: $\Delta$ (post-baseline)/baseline; ns, no significance; partial $\eta^{2}$ value for effect size.

\section{Discussion}

The present study aimed to investigate the effects of running and functional highintensity training on body composition, aerobic capacity, and muscle fitness. The primary finding was that high-intensity functional training was as effective as high-intensity interval running for aerobic capacity and body composition promotion in healthy inactive females, and moreover, it induced a significant improvement in muscle fitness. The validity of this finding is supported by the fact that the mean heart rate of all of the participants reached $75 \% \mathrm{VO}_{2}$ max or above throughout the intervention. Increases in resting heart rate were also detected after training in both groups.

\subsection{Body Composition}

Our findings that HIIT-R and HIIT-F had positive effects on body composition promotion regarding the reduction of the body fat percentage were consistent with other researchers. A previous study [49] showed improved body mass, BMI, and percent body fat among obese females after a total of 108 min HIIT-R. Similarly, previous research [50] found that HIIT-R was effective in reducing BMI and body fat percentage in overweight adults. Additionally, for individuals with normal BMI, body composition improved by decreasing fat mass and increasing lean mass after a 6 -week HIIT-R intervention [51].

Not surprisingly, body composition benefits were also found in other studies investigating HIFT. Improved body fat percentage was reported after a 5-week, thrice weekly HIFT intervention [25], and further studies have also indicated a beneficial influence of HIFT on body composition [52].

However, current research has indicated that body fat percentage was significantly improved after an eight-week HIFT, while body mass was unaltered [31]. Likewise, after 16 weeks of HIFT, a significant decrease in body fat percentage was observed with no changes in the body mass [26]. Previous HIIT-R studies have provided similar results [53,54]. These results are consistent with our findings that although body fat percentage was improved, body mass and BMI were not affected by the intervention. The improved body fat percentage may be explained by the significant increase in lean muscle mass ( $p=0.001$ for HIIT-R and $p=0.006$ HIIT-F) without significant changes in the body mass ( $p=0.064$ for HIIT-R and $p=0.051$ for HIIT-F). The non-significant change in BMI may be due to the following reasons: the insufficient exercise duration per session (2 min vs. 6-10 $\mathrm{min}$ ); the uncontrolled dietary intake during the intervention; and the characteristics of the participants regarding body weight. This suggestion has been highlighted in a recent systematic review [55] that indicated that for normal weight populations, low-volume HIIT is inefficient for body composition improvement. Furthermore, several studies have indicated that HIIT-R and HIFT have a more significant effect on weight loss or body fat loss among obese individuals [41,48-50].

Finally, no significant interaction effect was revealed for any body composition variables. This suggests that HIIT-R and HIIT-F were equally effective in the modulation of body fat percentage. 


\subsection{Aerobic Fitness}

$\mathrm{VO}_{2}$ max was assessed in the present study to estimate the effects of HIIT-R and HIIT-F protocols on aerobic fitness. Running-based HIIT has been shown to increase aerobic capacity in numerous previous investigations. Several studies have reported significant increases in $\mathrm{VO}_{2}$ max after HIT $[11,12,55]$. Furthermore, a systematic review also showed that HIIT was beneficial for aerobic fitness improvements among healthy young people [56]. Nevertheless, there has been no consensus on the effect of HIFT on aerobic capacity. Some studies investigating HIFT have shown an improvement in $\mathrm{VO}_{2} \max [35,52,57]$. On the contrary, recent research has only found aerobic capacity improvement in underweight and overweight boys, with no changes being found among normal weight people [39]. Similarly, no significant changes in $\mathrm{VO}_{2}$ max were found after a 6-week HIFT protocol [58,59].

In our study, participants from both the HIIT-R and HIIT-F groups experienced improvements in $\mathrm{VO}_{2} \max (17.1 \% \pm 5.6 \%$ and $12.7 \% \pm 6.7 \%$, respectively). In line with the magnitude of our results, an increase of $8 \%$ in the $\mathrm{VO}_{2}$ max was found after a low-volume HIFT [28]. It should be noted that in the current study, the enhanced $\mathrm{VO}_{2}$ max observed in the HIFT group was significantly higher than values recorded in previous studies. $\mathrm{VO}_{2}$ max has been reported to improve by $5 \%$ after a HIFT with no aerobic exercise [60]. Another study showed a moderate improvement in the $\mathrm{VO}_{2} \max$ of $6.3 \%$ [31]. In our study, the greater response of $\mathrm{VO}_{2}$ max to HIFT could be explained by the following reasons: firstly, improvements in $\mathrm{VO}_{2}$ max were related to the testing modality [61]. Cooper's 12 min run test demonstrated a systematic bias in favor of higher-scoring individuals [62]; secondly, this study used a longer duration (12 weeks vs. 6-8 weeks) for the implementation of functional exercises. Short or low-volume training reported no improvements in aerobic capacity, which was shown to require continuous training [14,63]. However, other investigations reported that the extent of improvement was not clearly related to training duration but to training intensity $[56,64]$. Therefore, further studies are required to investigate the effectiveness of the duration (work bouts/total work duration) and intensity on traininginduced aerobic capacity improvement; finally, the magnitude of the improvement in $\mathrm{VO}_{2}$ max can be attributed to the fatigue index, which was not measured in our study [11].

Although high-intensity running and functional training were both beneficial for aerobic capacity promotion, few studies have compared the effectiveness of these two exercise modalities in terms of aerobic capacity enhancement. In the current study, we controlled for the same intervention intensity and duration and found that surprisingly, there was no significant difference in terms of the changes in $\mathrm{VO}_{2}$ max between the HIIT- $\mathrm{R}$ and HIIT-F groups. It is worth noting that running showed higher oxygen consumption for the same intensity compared to other modalities [65]. Our findings were partially in line with a previous study [66] that indicated no significant differences in $\mathrm{VO}_{2}$ max promotion between high-intensity cycling and HIFT. The results from the present study illustrate that functional training is as effective as running for aerobic fitness improvement when performed at high intensity with the same volume and intensity.

\subsection{Muscle Performance}

Importantly, the repetition of sit-ups and the distance of the standing broad jump were significantly increased after HIIT-F, whereas both parameters remained unaltered in the HIIT-R group. Moreover, significant interaction effects were observed in terms of the effects on abdominal and lower limb strength and duration. Our finding is consistent with other HIFT studies. A significant increase in muscle performance after 6 weeks of HIFT was reported, whereas no increase was found in HIIT group using rowing as the exercise modality [27]. Significant improvements in lower body strength and power among patients and Army personnel were also evident [24,25]. Likewise, a study with female participants compared the effects of HIFT and endurance treadmill training on muscle fitness and demonstrated that sit-ups, chest presses, and push-ups improved by $64 \%, 207 \%$ and 135\%, respectively, in the HIFT group after 4 weeks of intervention [24]. 
It is worth noting that the number of flexed push-ups that was completed in the repetition exercise was unchanged in both groups. The unchanged results are in contrast with findings from other investigations. Findings from recent studies revealed increased upper body strength and endurance after functional training executed at a high intensity [24,26-28]. It was possible that the observed unvaried parameters were the consequence of insufficient movements during our functional training, which lacked upper body adaptations [26]. Additionally, the assessment methods used in the present study could have also induced unaltered results. Although the flexed push-ups had been modified for females and even though the participants were familiarized with testing procedures, the participants in the present study had no or little experience and were not familiar with this movement. Furthermore, they had no knowledge of specific strategies that could be used to maximize their performance.

The effects of HIFT on muscle performance varies across exercise design and test methods. HIIT significantly increases the proportion of type I fibers [67], while muscle adaptions are specific to the exercise modality. A previous study revealed that compared to high-intensity interval running, strength training with functional movements resulted in type I muscle fibers increasing in size and a higher percentage of type IIA muscle fibers [68]. In the present study, functional exercise was more effective in strengthening muscle power than running when both were performed at relatively the same high intensity and for the same duration. However, further studies are required to investigate the training-induced individual changes in the type and size of muscle fibers between participants. Additionally, the functional exercise design should consider the fitness of the participants to reduce muscle soreness, and a previous study reported no injuries using this methodology [31].

A general limitation in the HIFT investigation was the different types of functional exercises that were included. The results might be dissimilar if HIFT was performed with other combinations of movements. Furthermore, the results of our study came from a small sample size and a non-exercising control group was not used. Finally, dietary intake was not controlled during the intervention, and the total calories consumed were not calculated. In addition, the fatigue index was not measured during the aerobic test.

\section{Conclusions}

Twelve weeks of high-intensity training based on running or functional exercises were both effective in reducing body fat percentage and improving aerobic capacity among healthy inactive females. Relative to running-based high-intensity training, HIFT shows an equally effective alternative with more exercise enjoyment and much stronger adherence regarding body composition and aerobic fitness promotion. Additionally, HIFT resulted in greater muscle performance increases than running-based high-intensity training, after which no gains were observed in terms of muscle fitness.

HIFT with self-selected intensity represents an alternative to high-intensity interval running for eliminating exercise barriers for physical exertion. Furthermore, HIFT can be performed anywhere at any time, which limits the barriers of lacking time/money. Finally, HIFT reveals strong exercise adherence and more enjoyment among females. It may be helpful for individuals to promote physical activity and the associated benefits of a prolonged healthy lifestyle.

Author Contributions: Conceptualization, Y.L., H.D.W. and J.S.B.; methodology, H.D.W. and J.S.B.; software, Y.L.; validation, Y.L., H.D.W. and J.S.B.; formal analysis, Y.L.; investigation, Y.L.; resources, Y.L. and Q.W.; data curation, Y.L.; writing-original draft preparation, Y.L.; writing-review and editing, H.D.W. and J.S.B.; visualization, Y.L.; supervision, H.D.W. and J.S.B.; project administration, Y.L.; funding acquisition, Q.W. All authors have read and agreed to the published version of the manuscript. 
Funding: This research received no external funding.

Institutional Review Board Statement: The study was conducted according to the guidelines of the Declaration of Helsinki and was approved by the Institutional Review Board (or Ethics Committee) of Ningbo University (RAGH202103150366.8; 15 March 2021).

Informed Consent Statement: Informed consent was obtained from all subjects involved in the study.

Data Availability Statement: The data presented in this study are available upon request from the corresponding author. The data are not publicly available due to student privacy.

Acknowledgments: I would like to show my deepest gratitude to my supervisory team, Wiltshire and Professor Baker; they are respectable, responsible and resourceful scholars. They have provided me with valuable guidance in every stage of my research. Additionally, I would like to thank the researchers from Faculty of Sport Science, Ningbo University, for helping me to complete the assessment. Finally, many thanks to the participants who continued to exercise for their health.

Conflicts of Interest: The authors declare no conflict of interest.

\section{References}

1. Bermejo-Cantarero, A.; Álvarez-Bueno, C.; Martinez-Vizcaino, V.; García-Hermoso, A.; Torres-Costoso, A.I.; Sánchez-López, M. Association between physical activity, sedentary behavior, and fitness with health related quality of life in healthy children and adolescents: A protocol for a systematic review and meta-analysis. Medicine 2017, 96, e6407. [CrossRef]

2. Biddle, S.J.; Gorely, T.; Stensel, D.J. Health-enhancing physical activity and sedentary behaviour in children and adolescents. J. Sports Sci. 2004, 22, 679-701. [CrossRef]

3. Hallal, P.C.; Victora, C.G.; Azevedo, M.R.; Wells, J.C. Adolescent physical activity and health: A systematic review. Sports Med. 2006, 36, 1019-1030. [CrossRef]

4. Bull, F.C.; Al-Ansari, S.S.; Biddle, S.; Borodulin, K.; Buman, M.P.; Cardon, G.; Carty, C.; Chaput, J.P.; Chastin, S.; Chou, R.; et al. World Health Organization 2020 guidelines on physical activity and sedentary behaviour. Br. J. Sports Med. 2020, 54, $1451-1462$. [CrossRef]

5. Liguori, G.; Feito, Y.; Fountaine, C.; Roy, B. ACSM's Guidelines for Exercise Testing and Prescription, 11th ed.; Wolters Kluwer: Philadelphia, PA, USA, 2021.

6. Hallal, P.C.; Andersen, L.B.; Bull, F.C.; Guthold, R.; Haskell, W.; Ekelund, U. Global physical activity levels: Surveillance progress, pitfalls, and prospects. Lancet 2012, 380, 247-257. [CrossRef]

7. Lovell, G.P.; El Ansari, W.; Parker, J.K. Perceived exercise benefits and barriers of non-exercising female university students in the United Kingdom. Int. J. Environ. Res. Public Health 2010, 7, 784-798. [CrossRef]

8. Reichert, F.F.; Barros, A.J.; Domingues, M.R.; Hallal, P.C. The role of perceived personal barriers to engagement in leisure-time physical activity. Am. J. Public Health 2007, 97, 515-519. [CrossRef]

9. Gillen, J.B.; Gibala, M.J. Is high-intensity interval training a time-efficient exercise strategy to improve health and fitness? Appl. Physiol. Nutr. Metab. 2014, 39, 409-412. [CrossRef]

10. Laursen, P.B.; Jenkins, D.G. The scientific basis for high-intensity interval training: Optimising training programmes and maximising performance in highly trained endurance athletes. Sports Med. 2002, 32, 53-73. [CrossRef] [PubMed]

11. Astorino, T.A.; Allen, R.P.; Roberson, D.W.; Jurancich, M. Effect of high-intensity interval training on cardiovascular function, VO2max, and muscular force. J. Strength Cond. Res. 2012, 26, 138-145. [CrossRef] [PubMed]

12. Dias, K.A.; Ingul, C.B.; Tjønna, A.E.; Keating, S.E.; Gomersall, S.R.; Follestad, T.; Hosseini, M.S.; Hollekim-Strand, S.M.; Ro, T.B.; Haram, M.; et al. Effect of High-Intensity Interval Training on Fitness, Fat Mass and Cardiometabolic Biomarkers in Children with Obesity: A Randomised Controlled Trial. Sports Med. 2018, 48, 733-746. [CrossRef]

13. Milanović, Z.; Sporiš, G.; Weston, M. Effectiveness of High-Intensity Interval Training (HIT) and Continuous Endurance Training for VO2max Improvements: A Systematic Review and Meta-Analysis of Controlled Trials. Sports Med. 2015, 45, 1469-1481. [CrossRef]

14. Sultana, R.N.; Sabag, A.; Keating, S.E.; Johnson, N.A. The Effect of Low-Volume High-Intensity Interval Training on Body Composition and Cardiorespiratory Fitness: A Systematic Review and Meta-Analysis. Sports Med. 2019, 49, 1687-1721. [CrossRef]

15. Gaesser, G.A.; Angadi, S.S. High-intensity interval training for health and fitness: Can less be more? J. Appl. Physiol. 2011, 111, 1540-1541. [CrossRef] [PubMed]

16. Billat, L.V. Interval training for performance: A scientific and empirical practice. Special recommendations for middle- and long-distance running. Part I: Aerobic interval training. Sports Med. 2001, 31, 13-31. [CrossRef] [PubMed]

17. Bartlett, J.D.; Close, G.L.; MacLaren, D.P.; Gregson, W.; Drust, B.; Morton, J.P. High-intensity interval running is perceived to be more enjoyable than moderate-intensity continuous exercise: Implications for exercise adherence. J. Sports Sci. 2011, 29, 547-553. [CrossRef] 
18. Aaltonen, S.; Rottensteiner, M.; Kaprio, J.; Kujala, U.M. Motives for physical activity among active and inactive persons in their mid-30s. Scand. J. Med. Sci. Sports 2014, 24, 727-735. [CrossRef] [PubMed]

19. Parfitt, G.; Rose, E.A.; Burgess, W.M. The psychological and physiological responses of sedentary individuals to prescribed and preferred intensity exercise. Br. J. Health Psychol. 2006, 11, 39-53. [CrossRef]

20. Ekkekakis, P.; Parfitt, G.; Petruzzello, S.J. The pleasure and displeasure people feel when they exercise at different intensities: Decennial update and progress towards a tripartite rationale for exercise intensity prescription. Sports Med. 2011, 41, 641-671. [CrossRef] [PubMed]

21. Ekkekakis, P.; Hall, E.E.; Petruzzello, S.J. The relationship between exercise intensity and affective responses demystified: To crack the 40-year-old nut, replace the 40-year-old nutcracker! Ann. Behav. Med. 2008, 35, 136-149. [CrossRef]

22. Feito, Y.; Heinrich, K.M.; Butcher, S.J.; Poston, W.S.C. High-Intensity Functional Training (HIFT): Definition and Research Implications for Improved Fitness. Sports 2018, 6, 76. [CrossRef]

23. Haddock, C.K.; Poston, W.S.; Heinrich, K.M.; Jahnke, S.A.; Jitnarin, N. The Benefits of High-Intensity Functional Training Fitness Programs for Military Personnel. Mil. Med. 2016, 181, e1508-e1514. [CrossRef]

24. Heinrich, K.M.; Spencer, V.; Fehl, N.; Poston, W.S. Mission essential fitness: Comparison of functional circuit training to traditional Army physical training for active duty military. Mil. Med. 2012, 177, 1125-1130. [CrossRef] [PubMed]

25. Heinrich, K.M.; Becker, C.; Carlisle, T.; Gilmore, K.; Hauser, J.; Frye, J.; Harms, C.A. High-intensity functional training improves functional movement and body composition among cancer survivors: A pilot study. Eur. J. Cancer Care 2015, 24, 812-817. [CrossRef] [PubMed]

26. Feito, Y.; Hoffstetter, W.; Serafini, P.; Mangine, G. Changes in body composition, bone metabolism, strength, and skill-specific performance resulting from 16-weeks of HIFT. PLoS ONE 2018, 13, e0198324. [CrossRef] [PubMed]

27. Buckley, S.; Knapp, K.; Lackie, A.; Lewry, C.; Horvey, K.; Benko, C.; Trinh, J.; Butcher, S. Multimodal high-intensity interval training increases muscle function and metabolic performance in females. Appl. Physiol. Nutr. Metab. 2015, 40, 1157-1162. [CrossRef] [PubMed]

28. McRae, G.; Payne, A.; Zelt, J.G.; Scribbans, T.D.; Jung, M.E.; Little, J.P.; Gurd, B.J. Extremely low volume, whole-body aerobicresistance training improves aerobic fitness and muscular endurance in females. Appl. Physiol. Nutr. Metab. 2012, 37, 1124-1131. [CrossRef] [PubMed]

29. Fisher, J.; Sales, A.; Carlson, L.; Steele, J. A comparison of the motivational factors between CrossFit participants and other resistance exercise modalities: A pilot study. J. Sports Med. Phys. Fitness 2017, 57, 1227-1234. [CrossRef]

30. Heinrich, K.M.; Patel, P.M.; O’Neal, J.L.; Heinrich, B.S. High-intensity compared to moderate-intensity training for exercise initiation, enjoyment, adherence, and intentions: An intervention study. BMC Public Health 2014, 14, 789. [CrossRef]

31. Brisebois, M.F.; Rigby, B.R.; Nichols, D.L. Physiological and Fitness Adaptations after Eight Weeks of High-Intensity Functional Training in Physically Inactive Adults. Sports 2018, 6, 146. [CrossRef]

32. Armour, M.; Ee, C.C.; Naidoo, D.; Ayati, Z.; Chalmers, K.J.; Steel, K.A.; de Manincor, M.J.; Delshad, E. Exercise for dysmenorrhoea. Cochrane Database Syst. Rev. 2019, 9, Cd004142. [CrossRef]

33. Motahari-Tabari, N.; Shirvani, M.A.; Alipour, A. Comparison of the Effect of Stretching Exercises and Mefenamic Acid on the Reduction of Pain and Menstruation Characteristics in Primary Dysmenorrhea: A Randomized Clinical Trial. Oman Med. J. 2017, 32, 47-53. [CrossRef] [PubMed]

34. Li, H. Reliability and Validity of Three Wristbands in Running with Change of Direction. Master's Thesis, Shanghai University of Sports, Shanghai, China, 2021.

35. Menz, V.; Marterer, N.; Amin, S.B.; Faulhaber, M.; Hansen, A.B.; Lawley, J.S. Functional vs. Running Low-Volume High-Intensity Interval Training: Effects on VO(2)max and Muscular Endurance. J. Sports Sci. Med. 2019, 18, 497-504.

36. Kushner, R.F.; Gudivaka, R.; Schoeller, D.A. Clinical characteristics influencing bioelectrical impedance analysis measurements. Am. J. Clin. Nutr 1996, 64, 423s-427s. [CrossRef] [PubMed]

37. Safrit, M.J.; Glaucia Costa, M.; Hooper, L.M.; Patterson, P.; Ehlert, S.A. The validity generalization of distance run tests. Can. J. Sport Sci. 1988, 13, 188-196. [PubMed]

38. Cooper, K.H. A means of assessing maximal oxygen intake. Correlation between field and treadmill testing. Jama 1968, 203, 201-204. [CrossRef] [PubMed]

39. Laughlin, N.T.; Busk, P.L. Relationships between selected muscle endurance tasks and gender. J. Strength Cond. Res. 2007, 21, 400-404. [CrossRef] [PubMed]

40. Ruiz, J.R.; Castro-Pinero, J.; Espana-Romero, V.; Artero, E.G.; Ortega, F.B.; Cuenca, M.M.; Jimenez-Pavon, D.; Chillon, P.; GirelaRejon, M.J.; Mora, J.; et al. Field-based fitness assessment in young people: The ALPHA health-related fitness test battery for children and adolescents. Br. J. Sports Med. 2011, 45, 518-524. [CrossRef]

41. Sperlich, B.; Wallmann-Sperlich, B.; Zinner, C.; Von Stauffenberg, V.; Losert, H.; Holmberg, H.C. Functional High-Intensity Circuit Training Improves Body Composition, Peak Oxygen Uptake, Strength, and Alters Certain Dimensions of Quality of Life in Overweight Women. Front. Physiol. 2017, 8, 172. [CrossRef]

42. Bianco, A.; Lupo, C.; Alesi, M.; Spina, S.; Raccuglia, M.; Thomas, E.; Paoli, A.; Palma, A. The sit up test to exhaustion as a test for muscular endurance evaluation. Springerplus 2015, 4, 309. [CrossRef] 
43. Snarr, R.L.; Esco, M.R. Electromyographic comparison of traditional and suspension push-ups. J. Hum. Kinet 2013, 39, 75-83. [CrossRef]

44. Chen, W.; Hammond-Bennett, A.; Hypnar, A.; Mason, S. Health-related physical fitness and physical activity in elementary school students. BMC Public Health 2018, 18, 195. [CrossRef] [PubMed]

45. Krishnan, A.; Sharma, D.; Bhatt, M.; Dixit, A.; Pradeep, P. Comparison between Standing Broad Jump test and Wingate test for assessing lower limb anaerobic power in elite sportsmen. Med. J. Armed Forces India 2017, 73, 140-145. [CrossRef]

46. Baker, J.; Ramsbottom, R.; Hazeldine, R. Maximal shuttle running over $40 \mathrm{~m}$ as a measure of anaerobic performance. Br. J. Sports Med. 1993, 27, 228-232. [CrossRef] [PubMed]

47. Tabata, I.; Nishimura, K.; Kouzaki, M.; Hirai, Y.; Ogita, F.; Miyachi, M.; Yamamoto, K. Effects of moderate-intensity endurance and high-intensity intermittent training on anaerobic capacity and VO2max. Med. Sci. Sports Exerc. 1996, 28, 1327-1330. [CrossRef]

48. Domaradzki, J.; Cichy, I.; Rokita, A.; Popowczak, M. Effects of Tabata Training During Physical Education Classes on Body Composition, Aerobic Capacity, and Anaerobic Performance of Under-, Normal- and Overweight Adolescents. Int. J. Environ. Res. Public Health 2020, 17, 876. [CrossRef]

49. Racil, G.; Coquart, J.B.; Elmontassar, W.; Haddad, M.; Goebel, R.; Chaouachi, A.; Amri, M.; Chamari, K. Greater effects of highcompared with moderate-intensity interval training on cardio-metabolic variables, blood leptin concentration and ratings of perceived exertion in obese adolescent females. Biol. Sport 2016, 33, 145-152. [CrossRef]

50. Tjønna, A.E.; Stølen, T.O.; Bye, A.; Volden, M.; Slørdahl, S.A.; Odegård, R.; Skogvoll, E.; Wisløff, U. Aerobic interval training reduces cardiovascular risk factors more than a multitreatment approach in overweight adolescents. Clin. Sci. 2009, 116, 317-326. [CrossRef] [PubMed]

51. Macpherson, R.E.; Hazell, T.J.; Olver, T.D.; Paterson, D.H.; Lemon, P.W. Run sprint interval training improves aerobic performance but not maximal cardiac output. Med. Sci. Sports Exerc. 2011, 43, 115-122. [CrossRef] [PubMed]

52. Murawska-Cialowicz, E.; Wojna, J.; Zuwala-Jagiello, J. Crossfit training changes brain-derived neurotrophic factor and irisin levels at rest, after wingate and progressive tests, and improves aerobic capacity and body composition of young physically active men and women. J. Physiol. Pharmacol. 2015, 66, 811-821.

53. Buchan, D.S.; Ollis, S.; Young, J.D.; Cooper, S.M.; Shield, J.P.; Baker, J.S. High intensity interval running enhances measures of physical fitness but not metabolic measures of cardiovascular disease risk in healthy adolescents. BMC Public Health 2013, 13, 498. [CrossRef] [PubMed]

54. Weston, K.L.; Azevedo, L.B.; Bock, S.; Weston, M.; George, K.P.; Batterham, A.M. Effect of Novel, School-Based High-Intensity Interval Training (HIT) on Cardiometabolic Health in Adolescents: Project FFAB (Fun Fast Activity Blasts)—An Exploratory Controlled Before-And-After Trial. PLoS ONE 2016, 11, e0159116. [CrossRef]

55. Batacan, R.B., Jr.; Duncan, M.J.; Dalbo, V.J.; Tucker, P.S.; Fenning, A.S. Effects of high-intensity interval training on cardiometabolic health: A systematic review and meta-analysis of intervention studies. Br. J. Sports Med. 2017, 51, 494-503. [CrossRef]

56. Gist, N.H.; Fedewa, M.V.; Dishman, R.K.; Cureton, K.J. Sprint interval training effects on aerobic capacity: A systematic review and meta-analysis. Sports Med. 2014, 44, 269-279. [CrossRef]

57. Nieuwoudt, S.; Fealy, C.E.; Foucher, J.A.; Scelsi, A.R.; Malin, S.K.; Pagadala, M.; Rocco, M.; Burguera, B.; Kirwan, J.P. Functional high-intensity training improves pancreatic $\beta$-cell function in adults with type 2 diabetes. Am. J. Physiol. Endocrinol. Metab. 2017, 313, E314-E320. [CrossRef]

58. Crawford, D.A.; Drake, N.B.; Carper, M.J.; DeBlauw, J.; Heinrich, K.M. Are Changes in Physical Work Capacity Induced by High-Intensity Functional Training Related to Changes in Associated Physiologic Measures? Sports 2018, 6, 26. [CrossRef]

59. Sobrero, G.; Arnett, S.; Schafer, M.; Stone, W.; Tolbert, T.A.; Salyer-Funk, A.; Crandall, J.; Farley, L.B.; Brown, J.; Lyons, S.; et al. A Comparison of High Intensity Functional Training and Circuit Training on Health and Performance Variables in Women: A Pilot Study. Women Sport Phys. Act. J. 2017, 25, 1-10. [CrossRef]

60. Gettman, L.R.; Pollock, M.L. Circuit Weight Training: A Critical Review of Its Physiological Benefits. Phys. Sportsmed. 1981, 9 , 44-60. [CrossRef] [PubMed]

61. Magel, J.R.; Foglia, G.F.; McArdle, W.D.; Gutin, B.; Pechar, G.S.; Katch, F.I. Specificity of swim training on maximum oxygen uptake. J. Appl. Physiol. 1975, 38, 151-155. [CrossRef] [PubMed]

62. Penry, J.T.; Wilcox, A.R.; Yun, J. Validity and reliability analysis of Cooper's 12-minute run and the multistage shuttle run in healthy adults. J. Strength Cond. Res. 2011, 25, 597-605. [CrossRef] [PubMed]

63. Rodas, G.; Ventura, J.L.; Cadefau, J.A.; Cussó, R.; Parra, J. A short training programme for the rapid improvement of both aerobic and anaerobic metabolism. Eur. J. Appl. Physiol. 2000, 82, 480-486. [CrossRef]

64. Sloth, M.; Sloth, D.; Overgaard, K.; Dalgas, U. Effects of sprint interval training on VO2max and aerobic exercise performance: A systematic review and meta-analysis. Scand. J. Med. Sci. Sports 2013, 23, e341-e352. [CrossRef] [PubMed]

65. Viana, R.B.; de Lira, C.A.B.; Naves, J.P.A.; Coswig, V.S.; Del Vecchio, F.B.; Gentil, P. Tabata protocol: A review of its application, variations and outcomes. Clin. Physiol. Funct. Imaging 2019, 39, 1-8. [CrossRef] [PubMed]

66. Gist, N.H.; Freese, E.C.; Cureton, K.J. Comparison of responses to two high-intensity intermittent exercise protocols. J. Strength Cond. Res. 2014, 28, 3033-3040. [CrossRef] [PubMed] 
67. Simoneau, J.A.; Lortie, G.; Boulay, M.R.; Marcotte, M.; Thibault, M.C.; Bouchard, C. Human skeletal muscle fiber type alteration with high-intensity intermittent training. Eur. J. Appl. Physiol. Occup. Physiol. 1985, 54, 250-253. [CrossRef]

68. Jakobsen, M.D.; Sundstrup, E.; Randers, M.B.; Kjær, M.; Andersen, L.L.; Krustrup, P.; Aagaard, P. The effect of strength training, recreational soccer and running exercise on stretch-shortening cycle muscle performance during countermovement jumping. Hum. Mov. Sci. 2012, 31, 970-986. [CrossRef] [PubMed] 